\title{
Optimal receiver for quantum cryptography with two coherent states
}

\author{
Konrad Banaszek \\ Instytut Fizyki Teoretycznej, Uniwersytet Warszawski, Hoża 69, \\ PL-00-681 Warszawa, Poland
}

\begin{abstract}
A setup for discriminating between arbitrary two coherent states of a single light mode with the highest success rate allowed by quantum mechanics is presented. Its application to time-multiplexed quantum key distribution is discussed.
\end{abstract}

PACS numbers: 03.67.Dd, 42.50.-p, 03.65.Bz

From the conceptual point of view, the simplest quantum protocol for establishing a cryptographic key is a transmission of a random sequence of two non-orthogonal states [1]. What makes this protocol secure, is the impossibility of discriminating between these two states with certainty. Thus, any attempt of eavesdropping inevitably generates noise in the transmission channel, which can be detected by the communicating parties [2]. A straightforward optical realisation of this protocol is the use of two weak coherent states in an interferometric setup $[1,3]$.

In the two-state protocol, the cryptographic key is constructed from these events, for which the receiving party managed to identify unambiguously the obtained quantum state. As the two used states are non-orthogonal, this procedure can be successful only for a fraction of the sequence. In order to maximize capacity of the transmission channel, the recipient should employ a measurement with the smallest possible probability of yielding an inconclusive result. The general problem of optimal distinction between two non-orthogonal states has been studied by Ivanovic [4], Dieks [5], and Peres [6], who explicitly described a quantum measurement with the required property. This measurement has been demonstrated for polarisation states of a single photon [7], and its realisation for a pair of coherent states with opposite phases has been described [8].

The purpose of this note is to show that the optimal measurement discrim- 


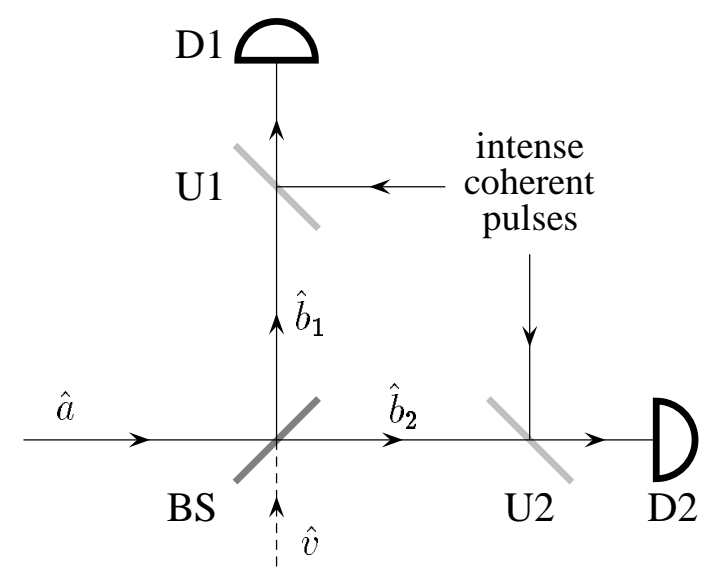

Fig. 1. Optical realization of the quantum measurement optimally discriminating between arbitrary two coherent states of a single light mode.

inating between arbitrary two coherent states of a single light mode can be implemented using a simple optical arrangement. I will present the quantum optical description of the setup and derive the positive operator-valued measure representing the measurement in the infinite-dimensional Hilbert space of the light mode used for communication. A possible application to timemultiplexed transmission using a single optical fibre will also be discussed.

In each run the sender (traditionally called Alice) generates a pulse in one of two coherent states: $\left|\alpha_{1}\right\rangle$ or $\left|\alpha_{2}\right\rangle$. The setup enabling the recipient (known as Bob) to distinguish optimally between these states is depicted in Fig. 1. First, the received pulse is split using a 50:50 beam splitter BS. The two outgoing fields, described by annihilation operators $\hat{b}_{1}$ and $\hat{b}_{2}$, are linear combinations of the incident fields:

$$
\left(\begin{array}{l}
\hat{b}_{1} \\
\hat{b}_{2}
\end{array}\right)=\frac{1}{\sqrt{2}}\left(\begin{array}{rr}
1 & 1 \\
1 & -1
\end{array}\right)\left(\begin{array}{l}
\hat{a} \\
\hat{v}
\end{array}\right)
$$

where $\hat{a}$ is the annihilation operator of the field received from Alice, and $\hat{v}$ describes the vacuum field entering through the second unused port of the beam splitter BS. Each of the outgoing fields $\hat{b}_{1}$ and $\hat{b}_{2}$ is transmitted via an unbalanced beam splitter (U1 or U2) with a very low reflection coefficient, and an auxiliary intense coherent pulse injected in the second port. In this regime, the beam splitters U1 and U2 effectively perform unitary displacement transformations on the transmitted fields, with the complex displacement parameters equal to the reflected amplitudes of the auxiliary coherent fields [9].

After transmission through the beam splitters U1 and U2, the fields are measured with the photodetectors D1 and D2, assumed to have unit quantum efficiency. Each of them yields one of two results: 0 when no photons were present, and 1 when at least one photon was registered. Quantum mechani- 
cally, the test for the presence of photons is described by a pair of projections on the vacuum state $|0\rangle\langle 0|$ and on the orthogonal subspace $\hat{I}-|0\rangle\langle 0|$. These projections can be expressed in terms of the modes $\hat{b}_{1}$ and $\hat{b}_{2}$ by applying appropriate displacement operators describing the action of the beam splitters U1 and U2. This transforms the vacuum states of the detector modes into coherent states $\left|\beta_{1}\right\rangle$ and $\left|\beta_{2}\right\rangle$ in the representation of the modes $\hat{b}_{1}$ and $\hat{b}_{2}$. Thus, the joint measurement performed by Bob is described by the following two-mode projections describing four possible outcomes:

$$
\begin{aligned}
& \hat{\mathcal{B}}_{00}=\left|\beta_{1}\right\rangle\left\langle\beta_{1}|\otimes| \beta_{2}\right\rangle\left\langle\beta_{2}\right| \\
& \hat{\mathcal{B}}_{01}=\left|\beta_{1}\right\rangle\left\langle\beta_{1}\right| \otimes\left(\hat{I}-\left|\beta_{2}\right\rangle\left\langle\beta_{2}\right|\right) \\
& \hat{\mathcal{B}}_{10}=\left(\hat{I}-\left|\beta_{1}\right\rangle\left\langle\beta_{1}\right|\right) \otimes\left|\beta_{2}\right\rangle\left\langle\beta_{2}\right| \\
& \hat{\mathcal{B}}_{11}=\left(\hat{I}-\left|\beta_{1}\right\rangle\left\langle\beta_{1}\right|\right) \otimes\left(\hat{I}-\left|\beta_{2}\right\rangle\left\langle\beta_{2}\right|\right)
\end{aligned}
$$

The parameters of Bob's setup are chosen such that

$$
\beta_{i}=\alpha_{i} / \sqrt{2}, \quad i=1,2 \text {. }
$$

Let us now find description of Bob's measurement in terms of the mode $\hat{a}$ used for communication. For this purpose one has to express the projection operators given in Eq. (2) in the representation of the modes $\hat{a}$ and $\hat{v}$, and then evaluate the expectation value over the vacuum mode $\hat{v}$. This yields a positive operator-valued measure

$$
\hat{\mathcal{A}}_{k l}=\left\langle 0_{v}\left|\hat{\mathcal{B}}_{k l}\right| 0_{v}\right\rangle, \quad k, l=0,1
$$

defined on the Hilbert space of the mode $\hat{a}$. The calculation described above can be easily performed with the help of the following normally ordered representation of projections on coherent states:

$$
\left|\beta_{i}\right\rangle\left\langle\beta_{i}\right|=: \exp \left[-\left(\hat{b}_{i}^{\dagger}-\beta_{i}^{*}\right)\left(\hat{b}-\beta_{i}\right)\right]:, \quad i=1,2 .
$$

This formula allows one to apply directly the transformation given in Eq. (1) and to eliminate the vacuum mode in a straightforward manner. The final result is

$$
\begin{aligned}
& \hat{\mathcal{A}}_{00}=: \hat{Q}_{1} \hat{Q}_{2}: \\
& \hat{\mathcal{A}}_{01}=: \hat{Q}_{1}:-: \hat{Q}_{1} \hat{Q}_{2}: \\
& \hat{\mathcal{A}}_{10}=: \hat{Q}_{2}:-: \hat{Q}_{1} \hat{Q}_{2}: \\
& \hat{\mathcal{A}}_{11}=\hat{I}-: \hat{Q}_{1}:-: \hat{Q}_{2}:+: \hat{Q}_{1} \hat{Q}_{2}:
\end{aligned}
$$


where, after using relation (3)

$$
\begin{aligned}
& \hat{Q}_{1}=\exp \left(-\frac{1}{2}\left(\hat{a}^{\dagger}-\alpha_{1}^{*}\right)\left(\hat{a}-\alpha_{1}\right)\right) \\
& \hat{Q}_{2}=\exp \left(-\frac{1}{2}\left(\hat{a}^{\dagger}-\alpha_{2}^{*}\right)\left(\hat{a}-\alpha_{2}\right)\right) .
\end{aligned}
$$

The probabilities of obtaining two results: 00 and 11 are independent of the state sent by Alice:

$$
\begin{aligned}
& \left\langle\alpha_{1}\left|\hat{\mathcal{A}}_{00}\right| \alpha_{1}\right\rangle=\left\langle\alpha_{2}\left|\hat{\mathcal{A}}_{00}\right| \alpha_{2}\right\rangle=\exp \left(-\left|\alpha_{1}-\alpha_{2}\right|^{2} / 2\right) \\
& \left\langle\alpha_{1}\left|\hat{\mathcal{A}}_{11}\right| \alpha_{1}\right\rangle=\left\langle\alpha_{2}\left|\hat{\mathcal{A}}_{11}\right| \alpha_{2}\right\rangle=0,
\end{aligned}
$$

whereas the remaining results may occur only for one of the received states:

$$
\begin{aligned}
& \left\langle\alpha_{1}\left|\hat{\mathcal{A}}_{01}\right| \alpha_{1}\right\rangle=\left\langle\alpha_{2}\left|\hat{\mathcal{A}}_{10}\right| \alpha_{2}\right\rangle=1-\exp \left(-\left|\alpha_{1}-\alpha_{2}\right|^{2} / 2\right) \\
& \left\langle\alpha_{2}\left|\hat{\mathcal{A}}_{01}\right| \alpha_{2}\right\rangle=\left\langle\alpha_{1}\left|\hat{\mathcal{A}}_{10}\right| \alpha_{1}\right\rangle=0 .
\end{aligned}
$$

Thus, the outcomes 01 and 10 enable Bob to identify uniquely the state sent by Alice, and these events can be used to establish a cryptographic key. From Eq. (8) it follows that the probability of obtaining an inconclusive result is $\exp \left(-\left|\alpha_{1}-\alpha_{2}\right|^{2} / 2\right)$, and equals the absolute value of the scalar product $\left|\left\langle\alpha_{1} \mid \alpha_{2}\right\rangle\right|$. This is the lowest rate allowed by quantum mechanics $[5,6]$, which acknowledges that the proposed setup optimally discriminates between the two coherent states used for secret communication.

The setup presented in Fig. 1 has a straightforward classical description. The coherent pulse received from Alice is split into equal parts and interfered with auxiliary pulses at beam splitters U1 and U2. These auxiliary pulses are selected such that for the two possible amplitudes of the pulse generated by Alice, destructive interference always takes place in one of the arms of the setup. Click on a detector means that destructive interference was not the case in the arm monitored by this detector, which allows Bob to identify uniquely the amplitude of the received pulse. Inconclusive result is when none of the detectors fired. In order to minimize frequency of such events, Bob should make the non-vanishing field detected by D1 or D2 as strong as possible. To achieve this, he has to avoid losses of the field received from Alice, and use low-reflection beam splitters U1 and U2.

In practice, the strong coherent pulses necessary to operate the receiver have to be provided by Alice in order to guarantee fixed phase difference between fields interfering at unbalanced beam splitters U1 and U2. Furthermore, it is 


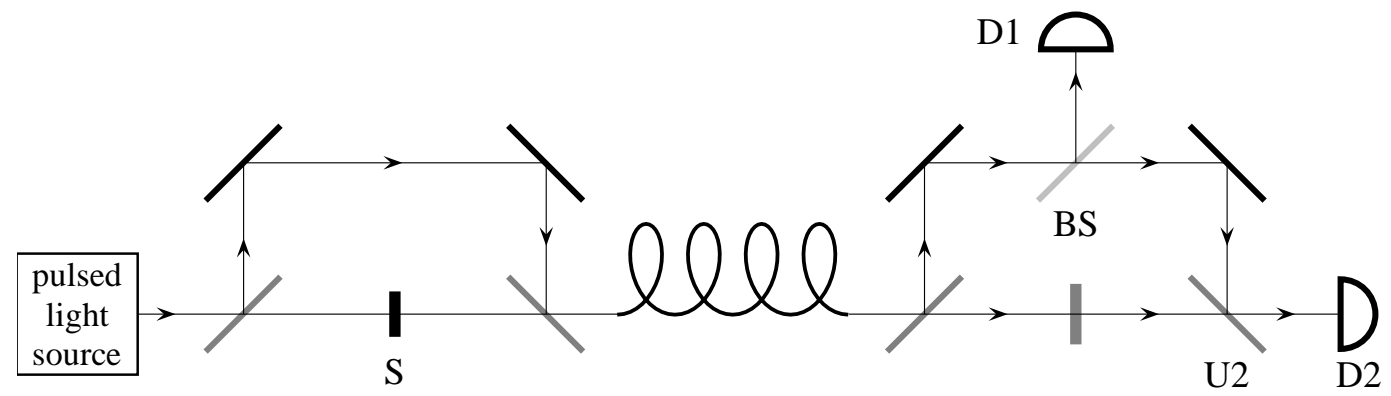

Fig. 2. A scheme for time-multiplexed quantum key distribution using the vacuum state and a weak coherent state.

convenient to send the auxiliary pulse along the same optical path as the signal one with a certain time separation. This enables stable transmission over large distances using optical fibers [3]. A setup for such time-multiplexed quantum key distribution is shown schematically in Fig. 2. Alice's transmitter is an unequal path interferometer with high-reflecting beam splitters. On the output of her device, Alice first generates either a faint coherent pulse or the vacuum by switching the shutter $\mathrm{S}$ placed in the shorter path of the interferometer. This weak quantum signal is followed by an auxiliary strong pulse travelling via the longer path. Assuming that all the unbalanced beam splitters in the setup have the same power transmission $T \ll 1$, the absolute value of the scalar product of the two states used by Alice is $\exp \left(-T^{2}|\gamma|^{2} / 2\right)$, where $\gamma$ is the amplitude of the pulse entering Alice's interferometer.

Bob's receiver is an analogous unequal path interferometer. The unambiguous measurement of Alice's state takes place when the signal pulse passes the longer path and overlaps with the auxiliary pulse transmitted via the two high-reflecting beam splitters. In the longer path, the beam splitter BS extracts about half of the signal to the detector D1. The remaining part is combined with the auxiliary pulse at the beam splitter U2. The amplitude and the phase of the auxiliary field is adjusted in the shorter path so that destructive interference occurs at the port monitored by the detector D2, when Alice send the faint coherent pulse. In this arrangement, the coherent state can trigger only D1, and the vacuum state can generate a click exclusively on D2.

In addition to detection events that yield unambiguous information on the received state, the detectors can also be triggered, with certain time separation, by the signal transmitted via the shorter path or the auxiliary pulse travelling via the longer path. The latter possibility is very likely due to large amplitude of this pulse. Therefore, appropriate timing between Alice and Bob is necessary in order to define the time window when the actual measurement takes place.

In order to make detection of two Alice's states equally probable (assuming that both the detectors are characterized by the same quantum efficiency $\eta$ ), 
the power transmission of the beam splitter BS should be $1 /(2-T)$, which for small $T$ is nearly $50 \%$. The success rate then equals $\exp \left[-\eta(1-T)^{2} T^{2}|\gamma|^{2} /(2-\right.$ $T)$ ], which for $T \ll 1$ and $\eta \rightarrow 1$ approaches the maximum value allowed by quantum mechanics.

Let us close this note with a comparison of classical and quantum descriptions of the presented setup. From the classical point of view, the reason for inconclusive results is that measured fields are too weak to trigger always one of the photodetectors. It may therefore seem that what primarily determines the failure rate is the characteristics of the photodetectors. However, quantum theory

of radiation reveals that the minimum failure rate is an intrinsic property of the two states of light to be distinguished, and depends solely on their scalar product. Another difference between classical and quantum descriptions is the role of vacuum fields. Classically, the vacuum field entering the input port of the beam splitter BS is irrelevant; in the quantum mechanical picture, it plays the role of an "ancilla" system, which extends the positive operator-valued measure $\hat{\mathcal{A}}_{k l}$ to the projective measure $\hat{\mathcal{B}}_{k l}$ on the two-mode Hilbert space.

\section{Acknowledgements}

The author wishes to acknowledge discussions with Prof. K. Wódkiewicz. This research was supported by the Polish KBN grant No. 2P03B 01315 and by Stypendium Krajowe dla Młodych Naukowców Fundacji na rzecz Nauki Polskiej.

\section{References}

[1] C. H. Bennett, Phys. Rev. Lett. 68 (1992) 3121.

[2] A. K. Ekert, B. Huttner, G. M. Palma, and A. Peres, Phys. Rev. A 50 (1994) 1047.

[3] R. J. Hughes et al., Contemp. Phys. 36 (1995) 149; S. J. D. Phoenix and P. D. Townsend, Contemp. Phys. 36 (1995) 165.

[4] I. D. Ivanovic, Phys. Lett. A 123 (1987) 257.

[5] D. Dieks, Phys. Lett. A 126 (1988) 303.

[6] A. Peres, Phys. Lett. A 128 (1988) 19.

[7] B. Huttner, A. Muller, J. D. Gautier, H. Zbinden, and N. Gisin, Phys. Rev. A 54 (1996) 3783. 
[8] B. Huttner, N. Imoto, N. Gisin, and T. Mor, Phys. Rev. A 51 (1995) 1863.

[9] K. Banaszek and K. Wódkiewicz, Phys. Rev. Lett. 76 (1996) 4344. 